\title{
La législation syndicale de Thatcher à Brown : menaces et opportunités pour les syndicats
}

Trade union legislation from Thatcher to Brown - threats and opportunities for trade unionism

John Mullen

\section{OpenEdition}

Journals

Édition électronique

URL : http://journals.openedition.org/rfcb/1140

DOI : $10.4000 /$ rfcb. 1140

ISSN : 2429-4373

Éditeur

CRECIB - Centre de recherche et d'études en civilisation britannique

Édition imprimée

Date de publication : 27 juillet 2009

Pagination : 73-85

ISBN : 978-2-9115-8028-4

ISSN : 0248-9015

\section{Référence électronique}

John Mullen, «La législation syndicale de Thatcher à Brown : menaces et opportunités pour les syndicats », Revue Française de Civilisation Britannique [En ligne], XV-2 | 2009, mis en ligne le 01 novembre 2016, consulté le 10 décembre 2020. URL : http://journals.openedition.org/rfcb/1140 DOI : https://doi.org/10.4000/rfcb. 1140

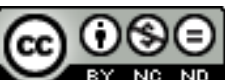

Revue française de civilisation britannique est mis à disposition selon les termes de la licence Creative Commons Attribution - Pas d'Utilisation Commerciale - Pas de Modification 4.0 International. 


\title{
La législation syndicale de Thatcher à Brown : menaces et opportunités pour les syndicats
}

\author{
John MULLEN \\ Université Paris 12
}

En 2007, les syndicats britanniques comptent 7,4 millions d'adhérents, chiffre qui n'a pas changé de façon significative depuis 12 ans. Ainsi, s'il n'est plus permis de voir le syndicalisme britannique en déclin continu depuis 1979, date de l'entrée en fonction du premier gouvernement Thatcher, on peut constater que le mouvement n'a pas encore su se remettre franchement de la période noire de 1980-1995, au cours de laquelle il a perdu 5 millions d'adhérents. La législation des gouvernements Thatcher-Major a été perçue comme un des facteurs principaux du déclin (« une politique ultralibérale qui anéantit la résistance syndicale » écrit Philippe Marlière) ${ }^{1}$. Cette contribution vise à retracer l'évolution des lois sur l'activité syndicale au Royaume-Uni depuis 1979, mais aussi à analyser les fondements idéologiques et les effets pratiques de ces lois. Les lois thatchériennes ont été conservées, presque en l'état, par les gouvernements New Labour qui ont suivi, symbole fort de leur rupture avec le passé du Parti travailliste. Toutefois, ces gouvernements ont voulu accorder de nouveaux droits aux syndicats en guise de compensation partielle, et afin de conserver le soutien politique et financier des organisations syndicales au Parti travailliste.

\section{Un nouveau conservatisme : l'arrivée au pouvoir de Margaret Thatcher}

L'une des priorités thatchériennes fut d'affaiblir le syndicalisme, mouvement prétendu illégitime qui atteignait l'esprit de l'entreprise, menaçait la santé économique du pays et était, selon les «thatchériens », le plus souvent contrôlé par une minorité de radicaux peu représentatifs. Le manifeste conservateur de 1979 adopte un ton ferme mais non dépourvu d'un sens de la diplomatie. Les syndicats y sont décrits comme facteurs agissant contre la cohésion sociale, mais un syndicalisme réformé y est considéré comme très utile. Il y est fait mention de syndicats «libres », libérés de l'influence d'une minorité malveillante: «des syndicats libres ne peuvent émerger que dans une société libre. Un mouvement syndical fort et responsable pourrait jouer un rôle important dans notre redressement économique ${ }^{2} \gg$.

\footnotetext{
1 «Un néotravaillisme très conservateur », Le Monde Diplomatique mai 2005

2 'Free trade unions can only flourish in a free society. A strong and responsible trade union movement could play a big part in our economic recovery.' Conservative Party Manifesto
} 
La faute y est largement présentée comme imputable au gouvernement travailliste :

Entre 1974 et 1976, le gouvernement travailliste a voté une série de lois sur les syndicats qui constitue une «Charte des extrémistes». Celle-ci a déséquilibré le rapport de force dans le dialogue social en faveur des syndicats, au détriment de la gestion responsable des entreprises. Elle a également favorisé des groupes de salariés agissant contre la volonté de leur direction syndicale ${ }^{3}$.

En effet, les gouvernements travaillistes avaient abrogé le Industrial Relations Act de 1971 et ses diverses dispositions qui visaient à réduire la capacité des syndiqués à faire grève. Ils avaient également cherché à encourager la négociation collective en mettant en place une agence gouvernementale (Arbitration and Conciliation Service, ACAS) dont le rôle était de renforcer les structures de dialogue social.

Dès 1979, Mme Thatcher essaya de limiter l'efficacité de l'action syndicale, tout en évitant de provoquer une réaction d'opposition massive de la part des syndicats, telle que celle qu'avait suscitée Edward Heath dans les années 1970. Dès 1980, l'Employment Act était votée. Puis, les Employment Acts de 1982, de 1988 et de 1990, le Trade Union Act de 1984 et le Trade Union Reform and Employment Rights Act de 1993 vinrent confirmer qu'une politique de laisser-faire dans le domaine des relations du travail n'était plus de mise pour les gouvernements conservateurs. Le grand nombre de lois adoptées témoigne de la tactique gradualiste choisie face à un puissant adversaire, fort de 12 millions d'adhérents.

La nouvelle législation fut accompagnée d'autres politiques antisyndicales. L'État, plus grand employeur du pays, dont les salariés étaient davantage syndiqués que dans le secteur privé, s'efforçait de donner l'exemple d'une nouvelle fermeté. Lors du conflit des mineurs en 1984-1985, toute la force de l'État fut utilisée contre leur syndicat, symbole des vieilles traditions du mouvement ouvrier. Et avec succès, la défaite des mineurs produisit une longue démoralisation du mouvement syndical. Lors d'un deuxième conflit, qui a fait couler beaucoup d'encre, au Government Communications Headquarters (GCHQ), le gouvernement interdit les syndicats dans des métiers civils proches de la structure militaire. Il s'agissait sans doute d'un «ballon d'essai », et l'intention d'interdire plus largement le syndicalisme dans une partie du secteur public était perceptible. Un conflit de plus de dix ans s'ensuivit, jusqu'à l'élection de $\mathrm{M}$. Blair qui va réintégrer les derniers grévistes et réaffirmer le droit de se syndiquer dans ces métiers. Dans cette affaire, le gouvernement conservateur n'a pas pu atteindre ses objectifs. Un troisième exemple de l'offensive antisyndicale de l'État employeur fut la décentralisation des négociations salariales

1979, disponible à www.conservative-party.net. Toutes les citations sont traduites par l'auteur.

3 'Between 1974 and 1976, Labour enacted a 'militants' charter' of trade union legislation. It tilted the balance of power in bargaining throughout industry away from responsible management and towards unions, and sometimes towards unofficial groups of workers acting in defiance of their official union leadership. 'Ibid. 
dans la fonction publique et, plus tard, la division de la fonction publique en entités autonomes (agencies) qui n'étaient plus soumises aux accords nationaux. L'efficacité de l'action syndicale en souffrit énormément. ${ }^{4}$

Les dispositions législatives des gouvernements Thatcher et Major peuvent être divisées en trois groupes: les obstacles posés à la grève, l'obligation de procéder à des élections des directions syndicales selon des modalités définies par le gouvernement et, enfin, une série de dispositions concernant l'activité « politique » des syndicats. Toutes ces dispositions seront introduites graduellement, et d'autres seront abandonnées car le rapport de force ne s'y prêtait pas.

\section{Faire obstacle à la grève}

Examinons d'abord les grèves. En 1980, le montant des allocations sociales consenties aux familles de grévistes est réduit, et l'immunité syndicale lors des grèves de solidarité est abolie. Les transformations du marché du travail et le développement de la sous-traitance rendent cette dernière restriction particulièrement contraignante: dans bien des secteurs, des salariés qui avaient précédemment fait partie de la même entreprise se trouvaient dispersés entre des structures juridiquement autonomes, et n'avaient donc plus le droit de faire ensemble une grève par solidarité. En 1982, les grèves politiques et de solidarité ne sont plus protégées par la loi. Les syndicats sont susceptibles d'être condamnés y compris à des dommages et intérêts s'ils appellent à une grève illégale. Ainsi, le Civil and Public Services Association, syndicat de fonctionnaires, a dû payer en 1988 une contravention de $£ 500000$ après avoir appelé à une grève de solidarité pour soutenir les salariés de la Santé ${ }^{5}$.

La législation connaît une pause au milieu des années 1980 (le conflit entre mineurs et gouvernement prend le relais) pour reprendre en 1990. Les entreprises peuvent désormais licencier les meneurs de grèves non officielles. La responsabilité légale des dirigeants syndicaux en cas de grève illégale est étendue : ils peuvent désormais être poursuivis pour dommages et intérêts s'ils ne dénoncent pas les grèves illégales par écrit. Ces dispositions indiquent la nouvelle confiance du gouvernement. Suite à la loi de 1993, des individus peuvent demander aux tribunaux des injonctions contre des grèves illégales. Un bureau gouvernemental pour la «protection contre les grèves illégales » est créé.

Le gouvernement Thatcher cherchait également à limiter l'efficacité des grèves déjà déclenchées. Or, la tradition du piquet de grève était forte dans le mouvement syndical anglais : dans les secteurs les plus fortement syndiqués, traverser un piquet de grève était considéré comme un déshonneur sans égal. Le plus souvent constitué d'une présence symbolique suffisant à faire rebrousser chemin à tout camionneur syndiqué, le piquet de grève pouvait, dans des conflits durs, devenir une manifestation de masse visant à empêcher physiquement l'entrée sur le lieu de travail de non-grévistes, comme à Saltley lors de la grève des mineurs de 1974. Pour

${ }^{4}$ Gregg P. and Machin S., Unions, the Demise of the Closed Shop and Wage Growth in the 1980s, Oxford Bulletin of Economics and Statistics, 54 (1992), pp. 53-72, passim.

${ }^{5}$ Mail on Sunday, 13 mars 1988. 
les thatchériens, cet outil syndical symbolisait le pouvoir de la masse contre le droit de l'individu de se rendre au travail. La loi de 1980 stipule que six grévistes au plus ont désormais le droit d'être présents sur un piquet de grève, qui ne peut se positionner que devant l'entreprise directement concernée et non chez un fournisseur ou un sous-traitant. Le Public Order Act de 1986 élargit la définition des crimes de « Riot» (émeute) et de «Violent disorder ». Désormais, il suffit qu'un non-gréviste se sente menacé pour rendre illégal un rassemblement, même en l'absence d'une menace avérée.

Ces lois ont parfois été utilisées. Lors de la grève des dockers de Liverpool, déclenchée en 1995, 400 grévistes furent licenciés car ils avaient organisé une grève illégale de solidarité. Les directions syndicales nationales refusèrent de lancer une campagne illégale de grèves pour leur défense. Il a souvent suffi à une entreprise de menacer de faire appel à la loi pour parvenir à contraindre une direction syndicale à renoncer à ses projets, ou pour démoraliser les partisans de la grève. Une enquête entreprise par la revue Labour Research en 1990 révèle que : «sur un tiers des lieux de travail, l'encadrement a menacé de recourir à la loi. Dans un cinquième de ceuxci, des grèves ou autres actions ont été annulées suite à ces menaces. Dans près d'un tiers des lieux de travail, les salariés ont déclaré qu'ils n'étaient pas prêts à faire grève à cause des dispositifs légaux ${ }^{6}$ ».

Pourtant, il n'est pas aisé de mesurer précisément l'efficacité de toutes ces mesures contre les grèves. Certes, le nombre de grèves a considérablement chuté pendant les années Thatcher-Major. Mais il est difficile de distinguer entre, d'une part, les effets d'un haut niveau de chômage et d'un déclin du syndicalisme dû à de nombreux autres facteurs (désindustrialisation, etc.) et, d'autre part, ceux provenant directement de la législation. Surtout, d'autres pays tels que la France ont également connu ce déclin brutal du nombre de journées de grèves au cours des années 1980 et 1990, sans pour autant être passés par une « révolution thatchérienne». D'ailleurs, même promulguées, les nouvelles lois furent assez rarement utilisées, surtout au cours des premières années, tant le rapport de forces semblait défavorable.

En tout et pour tout, pendant les dix premières années (1980-1990), ces lois n'ont conduit qu'une centaine de fois un syndicat devant un tribunal. Très peu de grévistes furent poursuivis au nom des lois sur les piquets de grève. Et nombreuses furent les grèves illégales n'ayant pas donné lieu à un appel à la loi. Il s'est produit des grèves de masse dans la fonction publique (en 1987, 50000 journées de grève en tout), contre l'embauche d'un dirigeant de parti fasciste et des grèves nationales de protestation contre l'interdiction du syndicalisme au GCHQ. Une étude sur les syndicats dans l'éducation a souligné que les employeurs peuvent ne pas vouloir appliquer la loi pour ne pas enflammer la situation ${ }^{\uparrow}$. On a observé des attitudes

${ }^{6}$ In one third of workplaces managers threatened to use the law. In one fifth of these, industrial action was called off because of these threats. In nearly one third of workplaces, workers said they were unwilling to strike for fear of the law. Labour Research, revue mensuelle du Labour Research Department, septembre 1990.

${ }^{7}$ J. ELGAR et R. SIMPSON, The Impact of the Law on Industrial Disputes in the 1980s, Report of a Survey of Education Authorities, Centre For Economic Performance, Discussion Paper No. 197, 1994, p. 47. 
similaires chez un grand nombre d'employeurs, tous secteurs confondus.

Néanmoins, les témoignages concordants d'employeurs et de syndicalistes montrent qu'il était devenu plus difficile d'organiser des grèves. Avant tout, la législation renforçait la position des dirigeants et des militants moins combatifs au sein de chaque syndicat. Prenons l'exemple de la grève dans l'imprimerie en 1983, chez Messenger Newspaper Group, qui n'a pas bénéficié de la traditionnelle forte solidarité syndicale dans le secteur. Il s'agit de la première grande victoire des lois thatchériennes, victoire qui a énormément contribué à la démoralisation du mouvement syndical. Autre exemple : au milieu des années 1990, lors d'une grève de masse, les postiers en Écosse débattirent de l'opportunité d'envoyer des piquets de grève pour demander la solidarité des centres de tri en Angleterre. Les lois fournirent l'argument clé aux syndiqués moins combatifs qui parvinrent à faire renoncer à ce projet. Aujourd'hui des piquets de grève dépassant le nombre autorisé restent courants, mais la menace de l'utilisation de la législation est toujours présente dès qu'un conflit s'envenime.

L'aile thatchérienne du Parti conservateur présentait les grèves comme déclenchées le plus souvent par une minorité radicale, contre la volonté d'une majorité silencieuse intimidée. La pratique syndicale de voter une grève à mains levée était considérée comme un outil de manipulation de la minorité. L'imposition de procédures de vote par bulletin secret devait donc réduire le nombre de conflits, et ces procédures furent rendues graduellement obligatoires. En 1980, le gouvernement n'osa pas encore avoir recours à la contrainte mais il encouragea l'organisation de scrutins avant le déclenchement d'une grève et proposait d'en couvrir les frais. À partir de 1984 (Trade Union Act) de telles consultations devinrent obligatoires. Après 1988, un vote séparé devait être organisé sur chaque lieu de travail (il n'était plus possible de prendre une décision nationale ou régionale). Enfin, à partir de 1993, le gouvernement Major imposa un préavis de 7 jours avant un vote sur la grève.

Selon le gouvernement, il s'agissait de promouvoir la démocratie au sein des syndicats. Les militants syndicaux défendaient pour leur part une conception de démocratie participative, où chacun devait justifier ses opinions devant ses collègues de travail. Ils donnaient pour argument que les scrutins bénéficiaient surtout aux employeurs, car les salariés sont isolés avec pour seule « aide » à leur décision celle des médias systématiquement anti-grévistes ${ }^{8}$. Certaines des dispositions prises avaient clairement pour objectif d'entamer la confiance des salariés. Par exemple la mention suivante est devenue obligatoire sur les bulletins de consultation à partir de 1988: " $\mathrm{Si}$ vous recourrez à la grève ou à d'autres actions revendicatives, vous pouvez vous trouver en situation de rupture de votre contrat de travail ${ }^{9} »$. Cette mention reste obligatoire même si l'action prévue ne peut pas constituer une rupture de contrat (par exemple un refus collectif d'heures supplémentaires - overtime ban). Au début de la discussion parlementaire sur ces mesures, le gouvernement avait même

${ }^{8}$ A. MANNING, An Economic Analysis of the Effects of Pre-strike Ballots, Londres : Centre for Economic Performance, London School of Economics and Political Science, 1992, p. 5.

9 'If you take part in a strike or other industrial action, you may be in breach of your contract of employment.' 
insisté pour que la réponse « Non » soit placée obligatoirement à gauche sur le bulletin de vote, afin que cela soit la première réponse lue par le salarié ${ }^{10}$ ! Pendant les années 1980, la revendication par le gouvernement d'un vote à bulletin secret avant une grève s'est transformée en une revendication d'un vote par voie postale. Pourtant le taux de participation est systématiquement plus faible dans de telles consultations. Il est permis de douter, donc, de la motivation démocratique du gouvernement.

Ces mesures ont-elles été couronnées de succès ? À nouveau, la réponse n'est pas facile. Manning affirme que «toute analyse de l'effet des consultations risque de relever de la spéculation ${ }^{11}$. Certains syndicats ont perdu beaucoup d'argent en contrevenant aux dispositions légales. En 1984, le Transport and General Workers Union dut payer une amende de $£ 200000$ pour défaut de scrutin avant une grève chez Austin Rover. Pendant la grève des mineurs de 1984-1985, tous les fonds du syndicat des mineurs furent saisis pour la même raison. Ces exemples ont fortement découragé les directions syndicales de passer outre les dispositions légales. Pourtant, l'obligation de respecter ces procédures n'a pas toujours eu l'effet escompté. L'idée thatchérienne selon laquelle les grèves étaient généralement imposées à des membres qui n'en voulaient pas a été démentie. Les scrutins secrets et postaux aboutissent presque toujours à soutenir la position de la direction du syndicat. Entre 1984 et 1987, sur 432 scrutins, $90 \%$ ont conclu par un soutien à la position officielle de la direction syndicale. Dix ans plus tard, le pourcentage est resté le même ${ }^{12}$.

Les lois ont néanmoins empêché de nombreuses grèves. En effet, le scrutin a tendance à remplacer la grève comme «thermomètre » informant l'employeur sur le niveau de combativité des travailleurs. Un vote en faveur d'une grève a souvent suffi pour amener l'employeur à faire de nouvelles concessions ${ }^{13}$. Le rapport produit par le Trades Union Congress en 1998 confirme cette analyse. La campagne conservatrice pour mettre en place des procédures étroitement surveillées avant le déclenchement des grèves a aussi servi à renforcer dans l'opinion publique (y compris auprès d'une minorité de syndiqués) la présentation des syndicats comme un problème, comme des structures freinant le progrès. Ceci a apporté des avantages peut-être inattendus aux projets gouvernementaux. À titre d'exemple, pendant la grève des mineurs, une bonne partie de la gauche politique ne soutenait pas les mineurs. La raison donnée fut souvent l'absence de scrutin avant le déclenchement de la grève. On peut affirmer qu'avant la mise en place de ces lois, personne n'aurait contesté la légitimité d'une telle grève, car elle jouissait du soutien évident de la majorité des mineurs.

\section{Contrôler l'action syndicale}

Du fait de leurs histoires et traditions très différentes, les syndicats avant la période Thatcher connaissaient une grande variété de modalités de sélection de leurs dirigeants : des collèges électoraux aux votes de délégués lors des congrès annuels,

${ }^{10}$ Labour Research Department (1989), Ballots on Industrial Action, Londres, LRD.

11 'any analysis of the effect of ballots is likely to remain largely speculative.' A. MANNING, An Economic Analysis of the Effects of Pre-strike Ballot, op.cit., p. 18.

${ }^{12}$ Focus on Balloting and Industrial Action, Trades Union Congress, 1998.

${ }^{13}$ W.A. BROWN, S. DEAKIN et P. RYAN, 'The Effects of British Industrial Relations Legislation, 1979-97', National Institute Economic Review, vol 161, N 1, 1997, pp. 69-83. 
en passant par le vote individuel des membres ou le vote bloqué par régions. Le gouvernement voyait en ces dirigeants une élite marxisante et pensait que de nouvelles modalités bénéficieraient aux modérés au sein des syndicats, tout en ayant l'avantage politique d'une « croisade pour la démocratie ». À nouveau, ce qui avait été présenté comme un encouragement en 1980 se transforma vite en contrainte. Dès 1982, l'élection d'un secrétaire général à vie devint illégale. À partir de 1984, tous les permanents devaient être élus tous les cinq ans à bulletin secret. À partir de 1988, tous les scrutins devaient être postaux et les membres des commissions exécutives devaient être élus même s'ils ne disposaient pas eux-mêmes du droit de vote dans ces instances.

Ces lois n'ont pas eu l'effet escompté. Entre 1988 et 1990, un seul secrétaire général fut limogé après l'introduction des nouvelles dispositions légales d'élection des dirigeants syndicaux. Nous n'avons pu trouver que deux exemples dans les années 1990, dans le syndicat des enseignants du supérieur (NATFHE) et dans le syndicat des journalistes (NUJ). Les nouvelles modalités n'ont profité systématiquement ni aux candidats « de gauche» ni aux candidats «de droite » au sein des syndicats. ${ }^{14}$ Cette inefficacité est en partie due au fait que, dans bien des syndicats, il n'existe pas de tendances organisées de gauche ou de droite qui proposent des candidats différenciés politiquement. Mais même dans les syndicats les plus politisés, l'effet n'a pas été de favoriser les candidats de droite, comme le montre l'élection en 2001 à bulletin secret et postal d'un candidat d'extrême gauche, Mark Serwotka, comme secrétaire général d'un des plus grands syndicats, le Public and Commercial Services. L'élection de plusieurs autres dirigeants qui déclaraient ouvertement leur adhésion à une philosophie fondée sur la « lutte des classes » tels que Jane Loftus (Communication Workers Union) ou Matt Wrack (Fire Brigades Union), confirme la superficialité de l'analyse thatchérienne des sources de la combativité syndicale.

En revanche, l'obligation de voter par correspondance a fait chuter les taux de participation. À titre d'exemple, celui que l'on constate pour l'élection du syndicat des salariés des transports, de $39 \%$ sous l'ancien système, a chuté à $17 \%$ avec le scrutin par correspondance. Le taux pour le syndicat des employés des impôts a chuté de $56 \%$ à $30,9 \%{ }^{15}$. Le système du closed shop, en vertu duquel le salarié est obligé d'adhérer au syndicat propre à l'entreprise avant d'être embauché, était honni par l'aile thatchérienne du Parti conservateur. Le combat fut compliqué, car de nombreux employeurs ne s'opposaient pas au closed shop, convaincus que l'institutionnalisation de la négociation avec un syndicat était préférable au risque de grèves sauvages. D'ailleurs, le closed shop constituait souvent un élément seulement d'un accord plus large entre syndicat et employeur, ce dernier bénéficiant de concessions syndicales en échange de l'introduction du monopole. En 1980, la loi octroya le droit à tout salarié, licencié pour avoir quitté le syndicat, de recourir au tribunal. Elle prévoit par ailleurs que la mise en place d'un monopole syndical ne puisse être effective qu'après un vote favorable des salariés à une majorité de $80 \%$. À partir de 1982, tout monopole syndical doit être confirmé par référendum tous les

${ }^{14}$ Roger UNDY et al, Managing the Unions. The Impact of Legislation on Trade Unions' Behaviour, Oxford: Clarendon Press, 1996, p. 190.

${ }^{15}$ Labour Research, mai 1990. 
cinq ans. En 1990, tout accord de monopole syndical devient illégal. Les premières lois sur les monopoles syndicaux eurent des résultats divers. Entre 1984 et 1986, 113 référendums se tinrent. Dans 88 cas, les $80 \%$ des voix nécessaires pour garder le monopole syndical furent atteints.

Nous l'avons vu, les grèves «politiques » ont été interdites. Mais d'autres actes politiques des syndicats inquiétaient également le gouvernement de Mme Thatcher, le financement du Parti travailliste n'étant pas des moindres. Plusieurs mesures furent prises pour s'attaquer à cet aspect de l'activité syndicale. Ces décisions étaient bien entendu marquées par la vision thatchérienne de directions syndicales gauchistes et illégitimes. À partir de 1984, toute activité politique d'un syndicat doit être financée par une caisse séparée - le «fonds politique » (political fund). La mise en place de tels fonds doit faire l'objet d'un référendum dans le syndicat tous les dix ans. Par ailleurs, la loi de 1988 prévoit que les congrès annuels des syndicats n'ont plus le pouvoir de décider de l'affiliation d'un syndicat à un parti politique (il s'agit toujours du Parti travailliste). Seul un référendum de tous les syndiqués peut entériner une telle décision. Le gouvernement a mené campagne pour tenter d'empêcher les syndicats de la fonction publique de mettre en place des fonds politiques. Avant un référendum organisé par un des syndicats de fonctionnaires, le ministère des Finances déclara : «les syndiqués devraient savoir que la mise en place de telles caisses sera perçue comme un non-respect du principe de neutralité politique de la fonction publique ${ }^{16} »$.

On peut dire que ces lois ont constitué une défaite pour Thatcher. Au cours des premières années, la totalité des 38 syndicats qui disposaient d'un fonds politique pour financer des campagnes électorales ou des candidats aux élections législatives ont voté à de larges majorités en faveur du maintien de ces fonds. ${ }^{17}$ D'ailleurs, en ce qui concerne le CPSA (syndicat de fonctionnaires), son fonds politique n'a été mis en place pour la première fois qu'en 1984. Nombre d'autres syndicats ont suivi cet exemple. Les directions syndicales et leurs bases ne semblent pas douter qu'une activité politique fasse partie du rôle normal d'un syndicat; le rêve d'un syndicalisme apolitique n'a pas été exaucé. En 1986, quatre syndicats, dont celui des impôts, ont créé pour la première fois de leur histoire un fonds politique. En 1987, cinq autres le créèrent, dont le syndicat de cadres de la fonction publique. En 1988, huit autres l'ont créé, dont le syndicat des employés municipaux et le Union of Democratic Mineworkers, une scission antigrève du syndicat des mineurs !

La mise en place des consultations sur les fonds politiques a provoqué un retour de bâton contre la politique de Thatcher. En effet, les militants de gauche dans plusieurs syndicats ont saisi cette opportunité pour faire campagne afin de convaincre les syndiqués de la nécessité de l'activité politique, et ont très souvent réussi. Cette mesure qui visait à dépolitiser le syndicalisme a plutôt repolitisé une série de syndicats. Les différentes lois des gouvernements conservateurs ont été assez efficaces, mais pas autant que ne l'avaient espéré leurs auteurs.

\footnotetext{
16 'Union members should realize that the creation of such funds will not be seen as in keeping with the political neutrality of a civil service' cité par le Morning Star, 8 février 1986.

${ }^{17}$ J. KELLY, Trade Unions and Socialist Politics, Londres: Verso, 1988, p. 280.
} 
Les gouvernements conservateurs de 1979 à 1997 et les gouvernements travaillistes depuis 1997 se sont tous intéressés au dispositif légal de la reconnaissance des syndicats par les entreprises. Dès 1980, les procédures de reconnaissance syndicale en vigueur sont abolies. En 1988, une commission «pour les droits des syndiqués » est établie afin de faciliter des recours de certains syndiqués contre leurs directions syndicales. La même année, il devient illégal de sanctionner un syndiqué qui a refusé de participer à une grève. En 1989, le nombre d'heures de délégation rémunérées aux représentants syndicaux est réduit. En 1993, on n'impose plus aux organismes publics tels que l'ACAS de « développer la négociation collective ». Cette approche changera radicalement lors de l'arrivée à Downing Street de Tony Blair.

La relative réussite de la campagne anti-syndicale des gouvernements conservateurs, malgré les échecs de certaines mesures législatives, fait souvent oublier le grand nombre de mesures qu'ils n'ont pas réussi à prendre. En 1988, Norman Fowler annonça sa volonté d'interdire purement et simplement les grèves dans les services publics «essentiels » ${ }^{18}$ mais le gouvernement n'osa pas donner suite à cette idée. Et la désyndicalisation du GCHQ, qui devait sans aucun doute n'être que la première d'une série, est restée un cas isolé. En 1988, il avait été question d'inclure dans la loi une mesure interdisant aux syndicats de la fonction publique l'affiliation au Parti travailliste, mais elle fut abandonnée.

Au-delà de l'impact concret de ces lois, il faut souligner l'importance de leur effet politique. Une série de mesures législatives « contre le pouvoir des syndicats » est parvenue à mettre cette question au centre du débat public, avec l'aide des grands médias largement gagnés aux thèses antisyndicales. Au sein d'une stratégie d'ensemble comprenant la privatisation et la dérégulation des services publics, et en général une domination plus forte du système de profit comme moteur moral et économique, ce positionnement politique a eu une importance centrale pour le gouvernement.

\section{Le Parti travailliste au pouvoir : l'action de Tony Blair et de Gordon Brown}

Au moment où nous écrivons, les conservateurs ne sont plus au pouvoir depuis plus de dix ans. Peut-on affirmer que les gouvernements de Blair et de Brown constituent, dans le domaine de la législation syndicale, un simple post-scriptum aux gouvernements conservateurs ? Certes, au centre du projet New Labour se trouvait la volonté de ne plus dépendre des structures syndicales ni en tant que parti ni en tant que gouvernement. La direction du Parti travailliste se revendiquait clairement d'une «troisième voie ", en faveur de la privatisation d'une grande partie du secteur public et d'une nouvelle manière de gérer les salariés de l'État comme les salariés d'une entreprise.

Dans le domaine législatif, une lecture de quelques extraits des manifestes travaillistes depuis 1979 éclaire la situation. Le manifeste de 1983 promet « nous

${ }^{18}$ Daily Telegraph, 18 avril 1988. 
abrogerons les lois sur l'emploi votées par les Conservateurs, lois qui sèment la discorde dans le pays $»^{19}$. En 1987, la promesse d'abroger les lois n'est pas répétée mais le parti s'engage comme suit : «nous nous assurerons que la loi protège la liberté des salariés et de leurs syndicats à organiser des actions revendicatives efficaces $»^{20}$. Le manifeste de 1992 indique un changement radical : «il n'y aura pas de retour vers la législation syndicale des années 1970. Les consultations avant les grèves, et pour les élections syndicales resteront en place. Il n'y aura pas de piquets de grève massifs, ni piquets de grève volants $»^{21}$. Et en 1997 le manifeste électoral indique que «les syndicats [doivent] comprendre qu'un gouvernement travailliste les traitera avec justice, mais ne leur accordera pas de faveurs $»^{22}$. Par ailleurs, il est affirmé que : "les élements clé de la législation syndicale des années 1980, concernant les consultations, les grèves et les piquets de grève, resteront en vigueur $»^{23}$. Pour ce qui est du manifeste travailliste pour les élections de 2001, les syndicats n'y sont quasiment pas évoqués dans le document, et aucun commentaire sur la législation syndicale n'est donné. La simple lecture de ces documents montre à quel point la nouvelle direction du Parti travailliste était convaincue que $\mathrm{M}$. Thatcher avait gagné la bataille de l'opinion publique sur la question syndicale.

Toutes les lois que nous avons énumérées ci-dessus sont donc restées en vigueur, avec quelques ajustements: le droit de l'employeur de licencier des grévistes a été restreint ; le délai après une consultation pendant laquelle un syndicat peut appeler à une grève est passé de quatre semaines à huit semaines; le poste gouvernemental de Commissaire pour les droits des syndiqués a été supprimé, et l'utilisation des «listes noires» de syndiqués par les employeurs est devenue illégale. Pourtant il y a eu d'importants changements. Une des conceptions fortes du thatchérisme était l'idée selon laquelle la négociation collective en soi constituait un élément perturbateur de la marche «normale » de la société. C'est pour cela que des institutions et procédures visant à encourager, voire à institutionnaliser la négociation collective ont été supprimées sous Mme Thatcher.

Le gouvernement Blair a une orientation tout à fait contraire. Il introduit dans l'Employment Relations Act en 1999 de nouvelles procédures de reconnaissance syndicale. Si une organisation syndicale peut démontrer que plus de la moitié des salariés sur un lieu de travail est adhérente, l'employeur est obligé de négocier avec ce syndicat. Si, par contre, moins de la moitié des salariés est adhérente, le syndicat peut demander à une commission spécialisée l'organisation d'une consultation. Une majorité relative suffit alors à faire reconnaître le syndicat : il suffit que $40 \%$ des salariés (et non des votants) s'expriment dans ce sens. Les syndiqués menant campagne pour une telle reconnaissance sur leur lieu de travail bénéficient d'une

\footnotetext{
19 'We will repeal the divisive tory 'employment laws"'

20 'We will ensure that the law guarantees the essential legal freedom of workers and their unions to organise effective industrial action.'

21 'There will be no return to the trade union legislation of the 1970s. Ballots before strikes and for union elections will stay. There will be no mass or flying pickets.'

22 'Unions accept they can get fairness but no favours from a Labour government...'

23 'The key elements of the trade union legislation of the 1980 s will stay - on ballots, picketing and industrial action.' Tous les manifestes sont disponibles en ligne à www.labourparty.org.uk/manifestos/
} 
protection contre le licenciement. Des représentants du patronat, comme le Institute of Directors, exprimèrent leur opposition à la nouvelle loi :

Les employeurs sont très troublés par les conséquences d'une reconnaissance syndicale obligatoire. Plutôt que se concentrer sur la gestion de leurs entreprises, les employeurs seront distraits par les négociations avec des représentants syndicaux concernant les salaires, les congés ou la formation. ${ }^{24}$

Il s'agit d'une opportunité octroyée aux syndicats de reconstruire leurs organisations. La loi de 1998 a contribué à la remontée relative du syndicalisme au Royaume Uni. Les syndicats ont pu en profiter pour mener des campagnes de recrutement modernes et dynamiques. Plus de 2500 accords ont été signés entre 1995 et 2003 concernant un peu plus d'un million de salariés, le plus souvent dans des entreprises où il y avait un fort mécontentement des salariés et donc la volonté de fonder une section syndicale. Ces nouveaux accords ont généralement permis aux syndicats d'obtenir rapidement des concessions de la part des employeurs : augmentations de salaire, nouveaux congés de maternité et de paternité, congés de formation, droit à davantage de choix dans les vêtements portés par les femmes au travail, réduction de la durée hebdomadaire de travail, etc. Les gains ont parfois été spectaculaires : à $\mathrm{CHC}$ helicopters les pilotes ont obtenu une augmentation de salaire de plus de $20 \%$ et à United Parcel Service une réduction de la semaine de travail de 55 heures à 40 heures $^{25}$. Les syndiqués espèrent que l'effet d'exemple permettra de recruter plus largement parmi les 5 millions de salariés sur des lieux de travail non syndiqués qui, selon un sondage récent, rejoindraient un syndicat s'ils en avaient la possibilitée ${ }^{26}$. Si certains employeurs hostiles ont été obligés de négocier avec les syndicats, d'autres, de meilleure grâce, ont cherché à établir un dialogue permanent, et ont autorisé, par exemple, des séances de recrutement par le syndicat lors des journées d'intégration de nouveaux salariés ${ }^{27}$.

Par la suite, d'autres dispositifs gouvernementaux viennent renforcer le rôle de la négociation sur le lieu de travail. Les nouveaux règlements sur l'information et la consultation des salariés, approuvés en 2004 et mis en place entre 2005 et 2008, prenant comme base les règlements européens, prévoient que les entreprises comptant plus de 50 salariés aient une obligation d'informer et de consulter des représentants des salariés concernant une large gamme de questions liées à la vie de l'entreprise (plans sociaux, restructurations, investissements...) Il s'agit de procédés similaires, mais plus légers, que les règlements couvrant les comités d'entreprise en France. Même les entreprises qui n'ont pas de section syndicale doivent mettre en

\footnotetext{
24 'Employers are perturbed about the consequences of compulsory trade union recognition. Rather than concentrating on running their firms, employers will be diverted into engaging in negotiations with union representatives over issues such as pay, hours, holidays and training.' Lettre ouverte à M Blair, Institute of Directors, Londres, 1998.

${ }^{25}$ G. GALL, The Fruits of Our Labour ? Outcomes of the new union recognition agreements, Trades Union Congress, Londres, 2003.

${ }^{26}$ TUC Reaching the Missing Millions, The report of the TUC's

Promoting Trade Unionism Task Group, Londres, 2001 ; GALL, op. cit., p. 4.

${ }^{27}$ Ibid. p. 13.
} 
place ces consultations, et l'élection de représentants du personnel à cette fin. Les syndicats espèrent qu'il s'agira d'une nouvelle opportunité pour recruter, et cela semble probable, même si les règlements permettent aussi - et ce n'est pas par hasard - des représentants non-syndiqués ${ }^{28}$.

Ainsi, les gouvernements New Labour ont eu une double attitude: conserver toutes les lois qui rendent la combativité syndicale et les grèves difficiles, mais encourager l'institutionnalisation d'un syndicalisme modéré. Le succès a été réel, et le soutien au gouvernement de la grande majorité des dirigeants syndicaux a été ferme. L'aile plus combative du syndicalisme anglais continue de tenter de faire reculer les lois thatchériennes. Le tout dernier acte de cette bataille a été celle du projet de loi Trade Union Freedom Bill, proposée par le Trades Union Congress en 2006, et porté devant le parlement non pas par le gouvernement travailliste mais par des députés partisans d'un syndicalisme combatif. Le projet de loi prévoyait une protection accrue contre le licenciement pour fait de grève, l'autorisation dans des circonstances limitées des grèves de solidarité ${ }^{29}$. Le projet de loi a fait l'objet d'une campagne syndicale, soutenue par des dizaines d'instances des divers syndicats, mais avec une mobilisation particulière des syndicats les plus militants. Il a peu de chances d'être adopté, mais la campagne sert de point d'appui pour demander des amendements éventuels aux lois existantes.

\section{Conclusion}

Les lois introduites par les gouvernements Thatcher et Major ont constitué une véritable menace pour le syndicalisme, et avant tout pour le syndicalisme combatif. Au milieu des années 1990, les grèves sont devenues plus difficiles à organiser et plus rares, le nombre de syndiqués a massivement baissé. Mais certaines de ces lois ont offert une opportunité à l'aile combative des syndicalistes : les lois sur les fonds politiques se sont retournées contre Mme Thatcher, les élections obligatoires des dirigeants ont permis une politisation de certains syndicats et les campagnes actives de recrutement par les syndicats sont devenues plus courantes. La bataille de l'opinion publique n'a pas été définitivement gagnée par les conservateurs; le consensus anti-syndical imaginé par Mme Thatcher n'a pas eu lieu. Des millions de salariés non-syndiqués persistent à voir les syndicats d'un oil sympathique et déclarent être prêts à rejoindre un syndicat si on leur en donnait la possibilité sur leur lieu de travail.

Les lois des gouvernements Thatcher et Major, introduites graduellement et en cherchant à éviter un conflit frontal, sont devenues une composante durable du paysage social en Grande Bretagne. Utilisant des sanctions financières contre les instances syndicales, et non des peines de prison prononcées contre des individus, elles ont été plus efficaces que celles de Heath pour affaiblir les syndicats et, avant tout, pour asseoir l'autorité des dirigeants syndicaux et des syndiqués moins combatifs. La législation a rendu le syndicalisme combatif plus difficile. Mais ce syndicalisme est loin d'avoir disparu. Des grèves sauvages illégales existent encore,

${ }^{28}$ TUC Guide to the Information and Consultation of Employees Regulations 2004, TUC 2005.

${ }^{29}$ www.tuc.org.uk. 
mais souvent elles ne sont ni répertoriées ni étudiées. À titre d'exemple, Le PDG de Gate Gourmet, entreprise qui fournit les repas pour les avions à l'aéroport de Heathrow, se plaignait que sept grèves sauvages aient été organisées entre 2002 et $2005^{30}$. L'entreprise a licencié 700 salariés en août 2005, du jour au lendemain, et une grève illégale de solidarité a arrêté l'essentiel des vols de l'aéroport. Lors de la vague de grèves à la poste en 2007 , les grèves sauvages, sans le soutien officiel de la direction du syndicat, ont constitué une bonne partie du total des journées de grève. L'élection de dirigeants syndicaux de la gauche radicale (voir ci-dessus) constitue un autre signe d'un potentiel radical durable. Enfin, les tactiques propres aux années 1970 (grands piquets de grève, grèves de solidarité illégales) font leur réapparition. En décembre 2004, lors de la grève à Alsthom, les piquets de grève comptaient plus d'une centaine de salariés.

Le Royaume Uni n'est pas un pays où les syndicats sont plus faibles qu'ailleurs. Le nombre de journées de grève en 2004-2006 y est bien plus élevé qu'en Allemagne ou au Portugal, même s'il est plus modeste qu'en Turquie ou en Espagne. Au moment où nous écrivons, une journée d'action dans la fonction publique, qui pourrait mobiliser plus d'un quart de million de grévistes, se prépare, et 100000 salariés dans les hôpitaux participent à un vote sur l'organisation d'une grève. La contribution de Tony Blair et de Gordon Brown a été de préférer l'implication des directions syndicales dans le «dialogue social » à une tentative de minimiser leur rôle. Le mouvement syndical britannique reste puissant, et sort lentement d'une longue période de relative démoralisation. Il est sujet à de nombreuses menaces, dont la législation n'est pas forcément la plus grave. La conviction de la plus grande partie des dirigeants syndicaux est qu'ils ne devraient pas affronter un gouvernement travailliste, la difficulté de trouver une nouvelle génération de délégués syndicaux pour prendre le relais des anciens, et une tendance de la part des salariés à voir dans le syndicat une simple source de « services » sont cités par les militants comme les principaux obstacles à une reconstruction durable d'un syndicalisme combatif ${ }^{31}$.

${ }^{30}$ Guardian 21 septembre 2005.

${ }^{31}$ GALL, op. cit. pp. $10 \& 18$ 